
internationales

vol. 30 - n² | 2014

Composer (avec) la frontière. Passages, parcours

migratoires et échanges sociaux

\title{
Franchir les dispositifs établis par Frontex. Coopérations policières transfrontalières et refoulements en mer Égée
}

Getting around Frontex Controls. Cross-border Police Cooperation and

Refoulement in the Aegean

Cruzar los controles establecidos por Frontex. La cooperación policial

transfronteriza y represiones en el Egeo

\section{Eva Ottavy et Olivier Clochard}

\section{(2) OpenEdition}

Journals

Édition électronique

URL : https://journals.openedition.org/remi/6931

DOI : 10.4000/remi.6931

ISSN : $1777-5418$

Éditeur

Université de Poitiers

Édition imprimée

Date de publication : 1 juin 2014

Pagination : 137-156

ISBN : 979-10-90426-22-1

ISSN : 0765-0752

Référence électronique

Eva Ottavy et Olivier Clochard, «Franchir les dispositifs établis par Frontex. Coopérations policières transfrontalières et refoulements en mer Égée », Revue européenne des migrations internationales [En ligne], vol. 30 - n² | 2014, mis en ligne le 01 juin 2017, consulté le 14 avril 2022. URL : http:// journals.openedition.org/remi/6931; DOI : https://doi.org/10.4000/remi.6931 


\section{Franchir les dispositifs établis par Frontex. Coopérations policières transfrontalières et refoulements en mer Égée}

\section{Eva Ottavy ${ }^{1}$ et Olivier Clochard ${ }^{2}$}

Depuis le début des années 2000, de nombreux responsables de gouvernements européens affirment que l'immigration dite clandestine serait en constante augmentation. Dans ces déclarations qui relèvent de clichés idéologiques, politiques ${ }^{3}$, voire policiers, il est régulièrement rappelé que les polices aux frontières des pays voisins ne participent pas suffisamment à la lutte contre l'immigration irrégulière et qu'en conséquence la coopération policière transfrontalière doit être renforcée. II ne fait pas de doute que ces argumentations - aussi diverses soient-elles - visent à légitimer le développement de nouvelles coopérations qui appellent à l'élaboration d'accords politiques ou à leur modification le cas échéant, à déployer de nouveaux dispositifs juridiques et techniques et à augmenter le nombre de policiers aux frontières. Pour rappel, en 2001, la politique des visas est devenue plus contraignante pour les ressortissants de pays tiers ${ }^{4}$, les compagnies de transports sont incluses dans la chaîne des contrôles migratoires avec des sanctions pécuniaires qui peuvent aller au-delà de 500000 euros pour les entreprises acheminant des personnes sans titre de voyage en règle. En 2004, un réseau d'officiers de liaison est officiellement mis en place ${ }^{5}$ pour lutter contre l'immigration " clandestine " dans les pays extérieurs à I'Union européenne (UE). Avec la communautarisation des politiques européennes, les coopérations policières n'ont donc cessé de se développer et la question du contrôle de l'immigration a pris une place croissante

\footnotetext{
1 Juriste, coordinatrice du réseau Migreurop, 21 ter rue Voltaire, 75011 Paris ; ottavy@ migreurop.org

2 Géographe, chargé de recherche CNRS, membre de Migreurop, MIGRINTER (CNRS UMR 7301 - Unniversité de Poitiers), MSHS, Bâtiment A5, 5 rue Théodore Lefebvre, 86000 Poitiers ; olivier.clochard@univ-poitiers.fr

3 « 1 million et demi de migrants sont en Libye, et ils sont susceptibles de gagner la rive sud de I'Union européenne " (Roberto Maroni, ministre de I'Intérieur italien, 2 mars 2011).

4 Le 15 mars 2001, les États membres ont adopté un règlement fixant une liste commune de près de 140 pays dont les ressortissants sont soumis à l'obligation de visa pour franchir les frontières extérieures de I'UE.
}

5 Règlement (CE) n³77/2004 du Conseil du 19 février 2004 relatif à la création d'un réseau d'officiers de liaison "Immigration ". 
dans ces diverses collaborations comme celles qui sont aujourd'hui sous la coupe de Frontex. Cette agence qui a été mise en place en 2004 pour coordonner les opérations de surveillance aux frontières extérieures de l'UE a pour principal objectif de lutter contre l'immigration irrégulière ${ }^{6}$. Mais quel est le mode de fonctionnement effectif de cette agence ? Comment des collaborations policières se mettent-elles en place aux frontières extérieures de I'Union européenne pour refouler plus facilement les étrangers démunis de documents en règle?

En s'intéressant au durcissement des contrôles migratoires aux frontières européennes qui se sont opérés au cours des dix dernières années, il s'agit tout d'abord de montrer le dispositif de l'agence Frontex pour être à même d'apprécier l'effort que les migrants doivent fournir pour le contourner lorsqu'ils ne disposent pas de document de voyage en règle. Pour bien comprendre l'émergence de cette agence au sein de I'UE nous adopterons un déroulement globalement chronologique. Puis dans un second temps, nous nous intéresserons aux situations de passages et de traversées - nombreuses et variées - qui s'opèrent en mer Égée, une des régions où depuis 2006 Frontex ne cesse de renforcer ses unités engendrant de nombreux refoulements vers la Turquie.

La méthodologie préalable à cet article repose sur un travail collectif d'associations et de chercheurs, mené au sein du réseau Migreurop et de la campagne Frontexit ${ }^{7}$ qui $s^{\prime}$ intéressent au respect des droits des migrants aux frontières extérieures de l'Union européenne et aux actions mises en place et coordonnées par l'agence Frontex. Dans cette optique, un important travail bibliographique a tout d'abord été effectué, notamment au sein de l'organisation britannique Statewatch ${ }^{8}$. Puis dans un second temps, nous sommes entrés en relation avec l'agence Frontex par le biais d'une dizaine de lettres dans lesquelles nous faisions part de nos interrogations sur des opérations mises en place ou des évènements dans lesquels Frontex semblait avoir un rôle. À chaque fois, par la signature de son directeur exécutif ou adjoint, l'agence a répondu relativement rapidement comme l'y oblige le règlement sur l'accès du public aux documents européens. Nous y avons vu un effort de transparence de la part de Frontex. Mais les éléments obtenus ne nous permettaient pas toujours de comprendre la compatibilité du mandat de l'institution avec les droits fondamentaux, ce qui nous a conduits à demander des compléments d'information par courrier et à organiser une mission d'enquête en mer Égée et à Évros, région frontalière avec laTurquie où des entretiens ${ }^{9}$ ont été menés.

6 Sur le site du Monde diplomatique, Jean-Marc Manach écrit qu'un « des membres de I'agence a reconnu, sous couvert d'anonymat, que "le travail de Frontex, c'est la lutte contre l'immigration illégale, pas le sauvetage en mer" ". Cf. Manach Jean-Marc (2014) Ces gens-là sont morts, ce ne sont plus des migrants, Le Monde diplomatique, 31 mars 2014, [en ligne]. URL : http://www.monde-diplomatique.fr/carnet/2014-03-31-morts-auxfrontieres

7 Voir le site de Frontexit (http://www.frontexit.org/fr/).

8 Voir le site de Statewatch (http://www.statewatch.org/observatories_files/frontex_observatory/index.html).

9 Ont participé à ces différentes missions qui ont eu lieu à I'automne 2013 : Eva Ottavy (Migreurop), Louise Tassin (Urmis/Migreurop), Laura Grant (FIDH), Anitta Kynsilehto (REMDH), Regina Mantanika (Urmis/Migreurop) et Marie Martin (Migreurop). 


\section{La mise en place de Frontex}

\section{Les opérations conjointes aux frontières extérieures : une idée née avec Schengen}

Avec la mise en place et l'élargissement de l'espace Schengen, les États membres de I'Union européenne (UE) et les pays associés à sa politique migratoire ont progressivement organisé des opérations policières conjointes visant à renforcer les contrôles migratoires. Si jusqu'à la fin des années 1980, le contrôle des frontières relevait du seul ressort des États souverains, les années 1990 furent en effet marquées par l'établissement de coopérations d'ordre bilatéral complétées par divers réseaux sémantiques où se sont élaborés de nouvelles doctrines, de nouveaux modes d'actions visant à renforcer les contrôles aux frontières (Bigo, 1996).

Puis la préoccupation croissante des États membres sur les questions relatives à la lutte contre l'immigration clandestine - liée en partie aux attentats de New York du 11 septembre 2001 - a accéléré la gestion " collective " des contrôles aux frontières extérieures de I'UE. Lors de la présidence belge, à l'automne 2001, il est en effet acté que les chefs de police des différents pays de I'UE - en charge de la surveillance des frontières - se rencontrent régulièrement ${ }^{10}$. En 2002, le SCIFA (Strategic Committee on Immigration, Frontiers and Asylum) est créé et la Commission européenne a pour objectif d'aller vers l'établissement d'une agence européenne visant à coordonner et renforcer le contrôle de l'immigration aux frontières extérieures ${ }^{11}$.

Par ailleurs, les pays formant les frontières orientales et méridionales de I'UE ont bien souvent souligné à la Commission européenne et aux autres États membres leurs souhaits de mutualiser les opérations de contrôles. De par leur situation géographique et la longueur de leurs frontières maritimes, l'Espagne, I'Italie et la Grèce disent être confrontées à des arrivées de migrants qu'elles jugent bien souvent comme " massives ", alors que les chiffres des personnes débarquées et interceptées rapportés au nombre de migrants arrivant en Europe sont à relativiser ${ }^{12}$. Ces mêmes États précisent également qu'ils effectuent les contrôles pour le compte de la plupart des pays européens. Face à ces situations migratoires, en mars 2003, la présidence grecque avait donc proposé quatre critères de partage de la charge du contrôle : la localisation géographique d'un pays, la nature de ses frontières, la pression migratoire et la qualité des contrôles. Mais cette proposition avait plutôt attisé les divisions entre les États membres. De plus, la Commission avait rappelé que chaque État membre était

10 Statewatch (2002) The European Border Guard: developing by Stealth?, Statewatch Bulletin, 12 (5), [en ligne]. URL : http://database.statewatch.org/article.asp?aid=6590

11 Communication de la Commíssion du 7 mai 2002 intitulée Vers une gestion intégrée des frontières extérieures des États membres et de I'Union européenne. Voir également Conseil européen de Séville (2002) Conclusions de la présidence (21 et 22 juin 2002), 42 p., [en ligne]. URL : http://www.consilium.europa.eu/ueDocs/cms_Data/docs/pressData/fr/ ec/72640.pdf

12 Au plus fort de la crise en 2008, les 160000 migrants interpellés à la frontière entre la Grèce et la Turquie représentent près de $0,8 \%$ des 19 millions de ressortissants de pays extérieurs à I'UE vivant dans les États membres. En 2011, le nombre de migrants interpellés est de l'ordre de 100000 (source: Commission européenne). 
garant financièrement des dispositifs mis en place aux frontières extérieures; le budget communautaire venant seulement en complément.

En octobre 2004, le règlement portant création de l'agence Frontex s'inscrit donc dans un contexte où les questions relatives aux contrôles des frontières extérieures sont guidées par des objectifs différents : pays européens du Sud et de l'Est qui souhaitent une mutualisation des contrôles et les autres États de I'UE qui demeurent plus timorés sur l'approche partagée de la surveillance à mettre en place aux frontières extérieures. En 2014, le développement de la coopération policière européenne demeure marqué par ces différends.

Par exemple la carte 1 (a et b) montre que des pays comme le Portugal, I'Italie, la Grèce, la Roumanie, la Pologne et la Lettonie, formant une grande partie de la frontière extérieure de I'UE, sont les principaux États qui mettent à disposition de Frontex des bateaux, des hélicoptères et des véhicules terrestres ${ }^{13}$. Ces dispositifs de surveillance comparés à ce dont disposait l'agence comme matériel en février 2010, soit vingt-six hélicoptères, vingt-deux avions légers et 113 navires (Casella Colombeau, Charles, Clochard et Rodier, 2010 : 6), indiquent également que les plans opérationnels se sont beaucoup développés sur le plan maritime. Le nombre de bateaux a triplé entre 2010 et 2014. Cette augmentation est liée à la conjonction de plusieurs facteurs : le déploiement de l'agence, les flux migratoires qui ont suivi le "Printemps arabe " et la volonté des gardefrontières des États membres de tenter de redorer une image ternie par les différents drames des boat people, et notamment celui du 3 octobre 2013 ayant entraîné la noyade de plus de 300 migrants au large de l'île de Lampedusa, et mettant surtout en lumière leur mandat relatif à la surveillance des frontières et bien moins le sauvetage des personnes en détresse. Enfin la réglementation de 2011 donne la possibilité à Frontex " d'acquérir, elle-même ou en copropriété avec un État membre, ou louer par crédit-bail des équipements techniques $" 14$ pour les opérations de contrôle, ouvrant ainsi la voie à d'éventuelles collaborations avec des acteurs privés et venant renforcer de fait les dispositifs existants. Concernant le nombre de policiers que l'agence a sous sa coupe (près de 2500 au total), la répartition est plus équilibrée, mais empreinte aussi d'un plus grand investissement des États membres, dont les frontières extérieures sont sujettes aux arrivées de migrants en situation " irrégulière ${ }^{15}$.

13 De son côté, I'Espagne a beaucoup investi dans le SIVE au sein du détroit de Gibraltar et des îles Canaries et dans les importants dispositifs (murs) entourant les présides de Ceuta et Melilla.

14 Article 7 du Parlement européen et du Conseil du 25 octobre 2011 modifiant le règlement (CE) n²007/2004 du Conseil portant création d'une Agence européenne pour la gestion de la coopération opérationnelle aux frontières extérieures des États membres de l'Union européenne.

15 Nous mettons des guillemets, car la convention de Genève relative au statut de réfugié du 28 juillet 1951 autorise les personnes en quête de protection à franchir une frontière sans document de voyage en règle. 


\section{Carte 1a : Mobilisation générale en mer Égée}
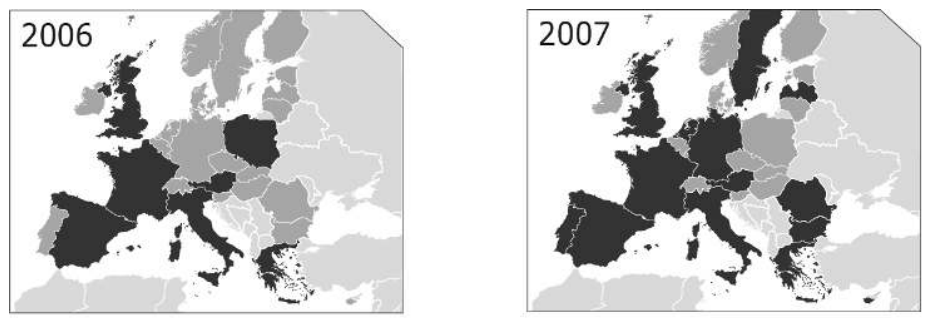

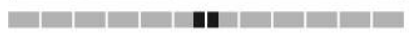
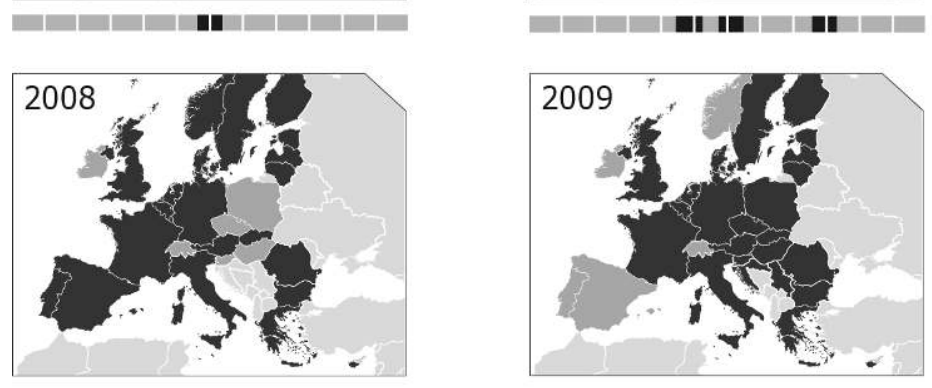

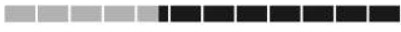
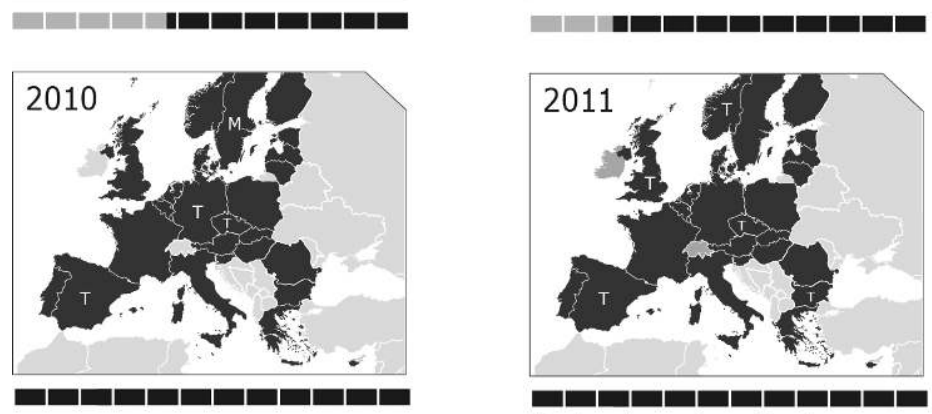

Membre observateur : Croatie
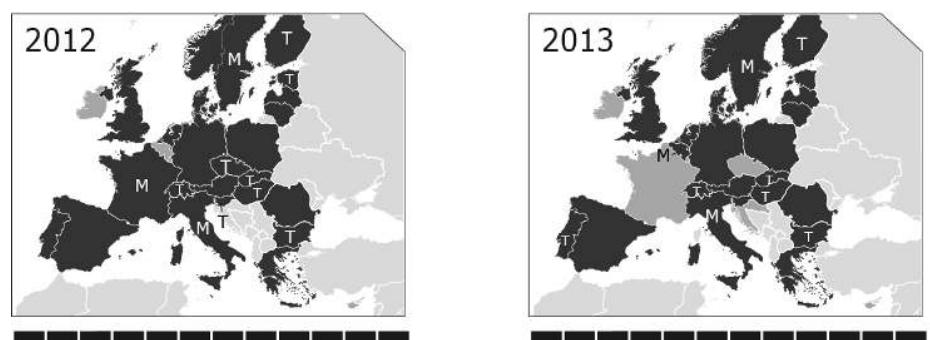

Membres observateurs : Croatie, Géorgie et Ukraine

- Période connue des interventions des unités de Frontex 1

${ }^{1}$ Pour chaque carte, le premier rectangle représente le mois de janvier et le dernier le mois de décembre.

Sources: Rapports annuels de l'agence Frontex.

Crédit : Olivier Clochard. 


\section{Carte 1b : Mobilisation générale en mer Égée}

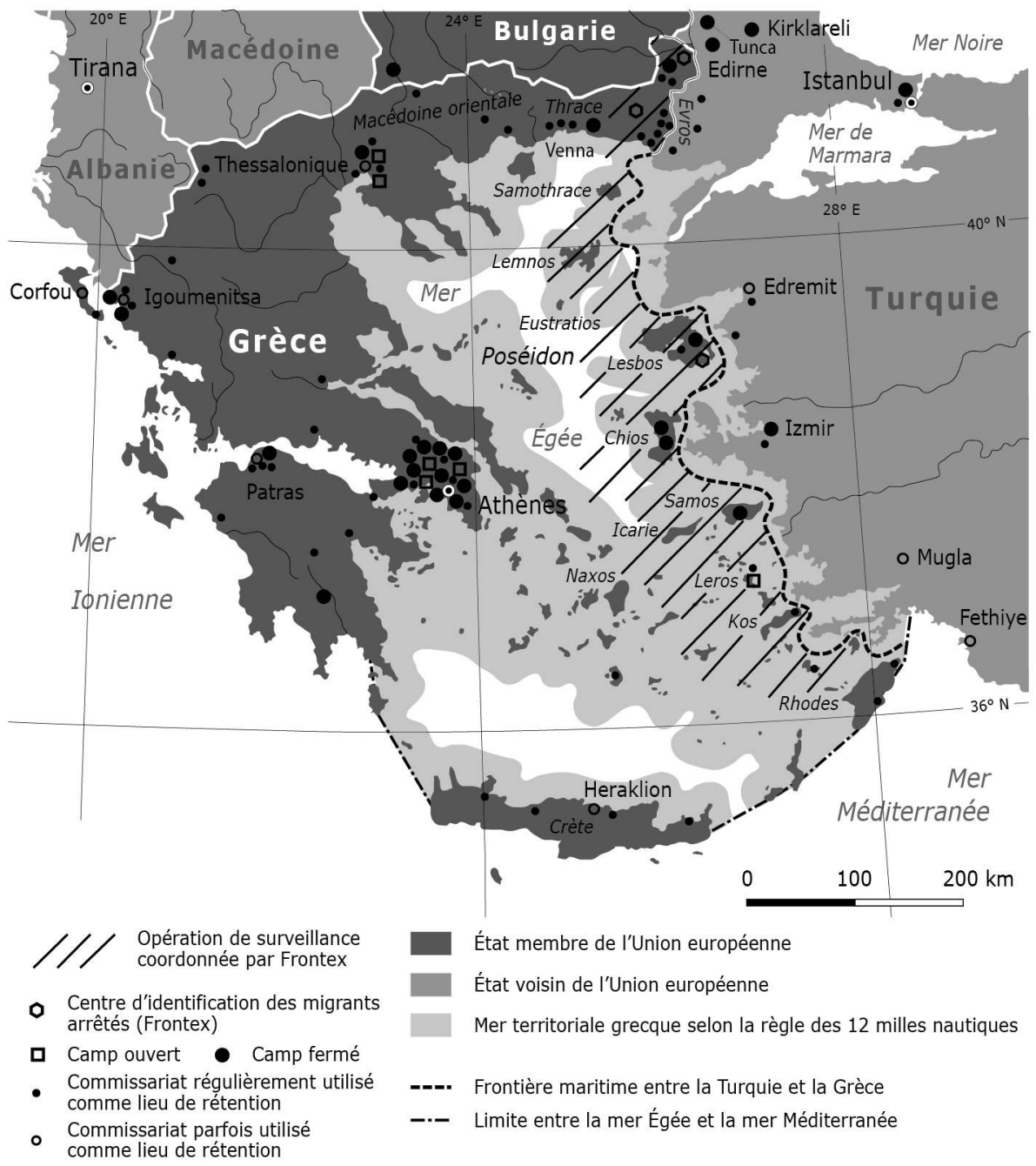

Sources: Associations Antigone, Diktio (Athènes) et HCA-RASP (Helsinki Citizens AssemblyRefugee Advocacy and Support Program/lstanbul) ; Rapports annuels de l'agence Frontex ; Wilson Andrew (1979) The Aegean Question, Adelphi Papers, 155 ; fond cartographique : Rekacewicz Philippe (1997) Prétentions grecques et turques sur la mer Égée, [en ligne].

URL : www.monde-diplomatique.fr/cartes/meregeemdv1997

Crédit : Olivier Clochard. 
Carte 2 : Du personnel et du matériel à la disposition de Frontex (2014)
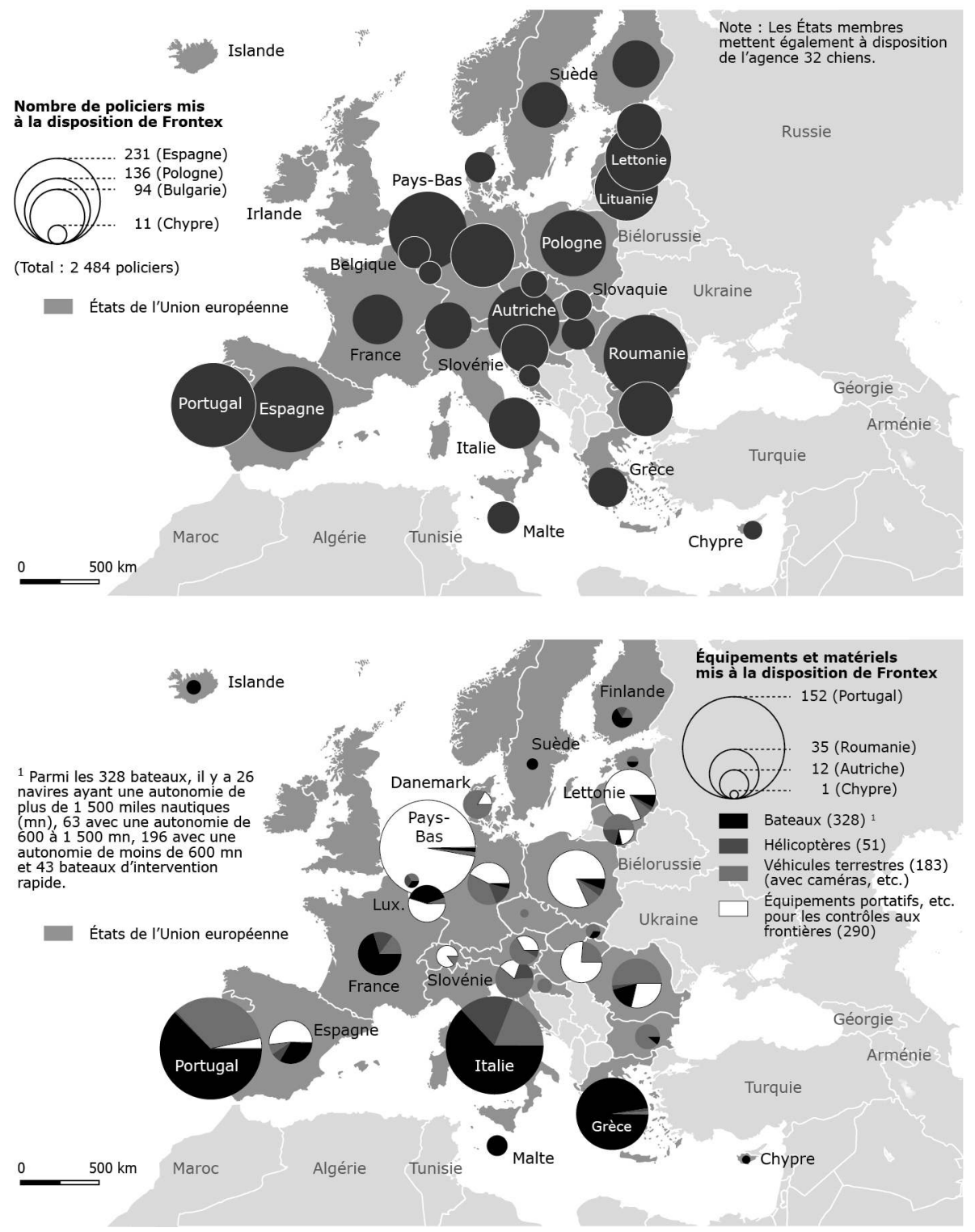

Source : Frontex (2014) Annual Information on the Commitments of the Member States to the European Border Guard Teams and the Technical Equipment Pool, 19 p.

Crédit : Olivier Clochard. 
Graphique 1 : Usage des équipements : prévisions pour l'année 2014

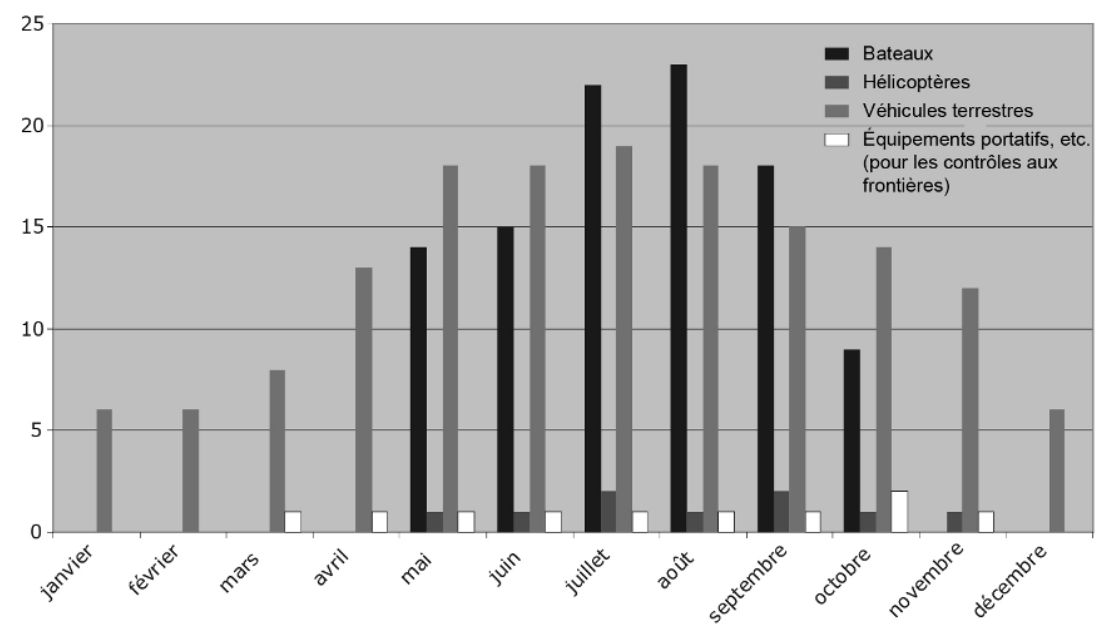

Source : Frontex (2014) Annual Information on the Commitments of the Member States to the European Border Guard Teams and the Technical Equipment Pool, 19 p.

Crédit : Olivier Clochard.

Depuis mai 2005, date à laquelle Frontex devient opérationnelle, le budget annuel de l'agence a fortement augmenté. Entre 2006 et 2013, il est passé de 19,2 à 94 millions d'euros, avec un pic de 118,2 millions d'euros en 2011 lié aux renforcements de la surveillance des flux migratoires au moment du "Printemps arabe ". Depuis 2008, Frontex est doté d'un budget plus important que les autres agences de I'UE comme Europol avec laquelle Frontex est amenée à collaborer. Cette évolution est liée à l'augmentation du nombre d'opérations qui ont également des durées plus conséquentes comme le montrent la carte 2 et le graphique 1. Mais si nous nous en tenons à ces seules lignes budgétaires, nous passons à côté d'autres aspects du déploiement de la coopération policière européenne en matière de contrôle des migrations. Par exemple, pour la Libye, un rapport non public du service européen pour l'action extérieure (SEAE) décrit qu'une mission EUBAM ${ }^{16}$ a été mise en place pour sécuriser les frontières de ce pays pour un montant de près de 140 millions d'euros. Les questions relatives à la lutte contre le terrorisme y sont évoquées, mais la gestion des migrations demeure également une préoccupation majeure. C'est ainsi que " Frontex a contribué aux activités préparatoires entreprises et coordonnées par [ce service]. [...] Et dans ce contexte, l'Agence a pris part à une mission d'information conduite [...] à Tripoli, dans l'optique de déterminer des actions possibles à mener en Libye pour aider les pouvoirs locaux à développer un système efficace de gestion des frontières" (Frontex, $2013: 7)^{17}$.

16 EUBAM, pour European Border Assistance Mission, s'appuie sur un ensemble d'acteurs institutionnels de I'Union européenne ayant pour objectif d'assister, voire de renforcer, la surveillance aux frontières des pays voisins de I'UE.

17 Voir également Fouteau Carine (2013) Le plan secret de I'Europe pour sécuriser la Libye, Mediapart, 22 novembre 2013. 


\section{La densification des réseaux et des communications de Frontex}

Pour renforcer son rôle, voire sa présence à l'extérieur de I'Union européenne, l'agence Frontex travaille donc de plus en plus étroitement avec les autorités de pays d'où proviennent ou par lesquels transitent de nombreux migrants et de nombreuses institutions internationales.

Concernant les pays avec lesquels l'agence collabore, il existe deux modalités de coopération. Dans un premier cas, Frontex peut s'appuyer sur une entente bilatérale entre un État membre et un pays tiers à l'image de l'accord signé entre I'Espagne et le Sénégal ou celui entre I'Italie et la Libye. Le cas échéant, les pays de I'UE sont contraints de faire figurer les modalités de collaboration de l'agence dans les accords bilatéraux qu'ils signent avec les pays tiers. Dans le second cas, c'est l'agence elle-même qui signe avec les autorités du pays concerné un accord qui a " uniquement trait à la gestion de la coopération opérationnelle ${ }^{18}$. Pour rappel il est prévu dans la législation européenne que les députés du Parlement européen (PE) puissent exercer un contrôle sur les accords internationaux, or pour ceux signés par Frontex et un ministère d'un pays tiers, ils en sont seulement informés. Ce point fait l'objet d'un différend important entre Frontex, certains représentants parlementaires (PE) et les associations de défense des droits des migrants, ces derniers estimant que le Parlement européen devrait avoir un droit de vote sur ces accords qu'ils considèrent comme internationaux. Mais l'agence ne cesse de rappeler que ce sont des accords techniques entre polices et non des accords politiques entre I'Union européenne et les pays tiers $^{19}$.

Au regard des différents accords que l'agence a signés, deux périodes se distinguent : une première entre 2006 et 2009 où sont concernés I'ensemble des pays des Balkans - à l'exception du Kosovo - et ceux situés à la frontière orientale de I'UE (Ukraine, Moldavie et Biélorussie), et une seconde période qui souligne la préoccupation de Frontex à l'égard des migrations en provenance d'Afrique et de Turquie. Pour l'Afrique, cela se traduit par la signature d'accords avec le Cap-Vert et le Nigéria ainsi que la mise en place de négociations avec des pays du pourtour méditerranéen (Libye, Tunisie, Maroc) et de la côte atlantique (Mauritanie, Sénégal, Gambie, Guinée-Bissau et Guinée-Conakry). Pour renforcer la surveillance des migrations en provenance de Turquie, un bureau régional a tout d'abord été mis en place dans le port du Pirée (Athènes) le 1er octobre 2010 afin d'être au plus près des opérations que l'agence coordonne en mer Égée et dans la région d'Évros, puis de nouveaux accords ont été signés avec I'Arménie, la Turquie et l'Azerbaïdjan venant compléter celui qui avait été scellé avec la Géorgie en 2008. La liste de ces pays témoigne de la mise en place d'un cordon sécuritaire autour de I'Union européenne, une sorte de "limes" européen (Migreurop, 2012 : 49).

Comme la convention d'application des accords de Schengen en son temps (1990), ces divers accords " introduisent dans les pratiques de coopérations [policières] un degré de formalisation et d'institutionnalisation supérieur "

18 Article 14 du règlement (UE) $n^{\circ} 1168 / 2011$.

19 Selon l'article 218TFUE, le contrôle démocratique du Parlement européen est prévu pour tout accord passé entre I'Union européenne et un pays tiers. 
(Maguer, 2002) visant à ce que l'ensemble des acteurs publics du contrôle frontalier participent aux opérations de l'agence. Pour faciliter ce processus, depuis 2011, Frontex peut déployer dans les pays extérieurs à I'UE ses propres officiers de liaisons "immigration " dont les actions vont venir renforcer celles des officiers de liaisons des États membres qui y travaillent en réseau depuis 2004. L'action de ces fonctionnaires est " de contribuer à la prévention de l'immigration illégale, à la lutte contre ce phénomène et au retour des migrants illégaux $\|^{20}$. Dans ce processus d'externalisation des contrôles migratoires, leur rôle a donc vocation à s'étendre, que ce soit en matière de récolte d'informations relatives à l'immigration dite irrégulière ou en matière de lancement et financement de projets d'assistance technique en vue de déployer de nouveaux moyens de contrôle dans les pays extérieurs à I'Union européenne (Casella Colombeau et Clochard, 2012 : 46). En contrepartie, des agents policiers de pays tiers peuvent être invités en tant qu'observateurs à participer aux opérations de l'agence aux frontières de l'UE (cf. Carte 2). Les officiers de Frontex assurent ainsi " I'interface " (Bigo, $1996: 29)$ entre diverses polices nationales, les douanes, des hauts fonctionnaires, etc. et renforcent le développement des relations transfrontalières et l'internationalisation de la surveillance des flux migratoires en accédant à de nouveaux territoires d'action ${ }^{21}$.

Parmi les potentiels collaborateurs référencés dans le règlement de l'agence, Frontex fait également appel à une multitude d'acteurs qui de par leurs actions, souligne la volonté de l'agence d'être informée de l'évolution des situations migratoires sur l'ensemble des frontières extérieures de I'UE. C'est ainsi qu'en 2008 des accords ont été conclus avec Europol et l'Agence européenne pour la sécurité maritime (EMSA) ${ }^{22}$. Frontex a aussi des liens étroits avec le Forum européen pour la recherche et l'innovation en matière de sécurité, le Centre international pour le développement des politiques migratoires (ICMPD) ${ }^{23}$, des institutions qui proposent et développent des aspects principalement sécuritaires de la gestion des frontières. Avec ces accords, il s'agit donc de légaliser le rôle de ces différents organismes dans la lutte contre l'immigration clandestine. En conséquence, les modalités des contrôles migratoires mises en place aux frontières de I'UE reposent de plus en plus sur des structures en archipel (Bigo, 1996 : 339) : soit un réseau d'administrations où les polices nationales chargées de la surveillance des frontières, les douanes, les officiers de liaison " immigration " en poste dans des pays étrangers, les consulats qui délivrent les visas ainsi que les compagnies de transport ou des sociétés privées de surveillance viennent s'adjoindre aux actions mises en place par Frontex. À cela s'ajoutent

\footnotetext{
20 Article 14 du règlement (UE) $n^{\circ} 1168 / 2011$

21 À propos de la coopération transfrontalière dans la Manche, Sheptycki (1997) souligne que " les officiers de liaison furent le moteur de l'échange d'informations entre les polices des frontières concernées ").

22 D'autres accords ont été passés avec le groupe opérationnel des chefs de polices de I'UE, le groupe de coopération douanière (GCD), I'Agence communautaire pour le contrôle des pêches, I'Agence des Nations unies contre la drogue et le crime, etc. (cf. Migreurop, 2012 : 53).

23 Par exemple, Frontex travaille avec le ICMPD (International Centre for Migration Policy Development) sur un projet intitulé Support dialogue on Mediterranean Transit Migration, programme auquel Europol est associé. La collaboration avec plusieurs de ces organisations internationales peut être considérée comme une valeur ajoutée à la lutte contre l'immigration " irrégulière ".
} 
les réseaux informatiques comme le SIS (Système d'Information Schengen), concernant notamment les personnes interdites de séjour, et le dispositif Eurosur que I'UE a progressivement développé depuis 2008, permettant aux autorités des pays membres d'échanger rapidement des informations opérationnelles et de coopérer entre elles ainsi qu'avec l'agence Frontex et les pays voisins de I'UE.

Enfin un forum consultatif a été créé " par l'Agence pour assister le directeur exécutif et le conseil d'administration dans les matières concernant les droits fondamentaux ${ }^{24}$. Les organismes internationaux ${ }^{25}$ présents au sein de ce forum relèvent d'institutions onusiennes, européennes ou interétatiques comme I'OIM, voire du monde des ONG. Sans impliquer systématiquement une coordination effective entre l'ensemble de ces organisations, les différents accords passés avec l'agence les rendent désormais, directement ou indirectement et à des titres différents, acteurs des opérations mises en place par Frontex.

\section{La question des droits fondamentaux}

Dans le règlement de 2004 relatif à la création de Frontex, les garanties juridiques relatives aux droits des migrants - comme le respect effectif du droit d'asile lors des opérations coordonnées par l'agence - ne sont pas clairement établies. Par exemple, s'il est indiqué que l'agence « respecte les droits fondamentaux et observe les principes reconnus par [...] [le] traité sur I'Union européenne et réaffirmés par la charte des droits fondamentaux de I'Union européenne ${ }^{26}$, il n'est mentionné aucun terme qui fait référence aux questions relatives à I'asile ou au non-refoulement des migrants interceptés, tel qu'il est défini dans l'article 33 de la convention de Genève de 1951. Néanmoins dans les rapports annuels de l'agence, il est mentionné qu'en 2007 " 130000 ressortissants de pays tiers ont été refoulés de I'UE tandis que le rapport de l'année 2008 fait état de 140000 refus d'entrée. Dans ces deux rapports, la présence éventuelle, parmi ces refoulés, de demandeurs d'asile potentiels ou personnes vulnérables (mineurs isolés, etc.) n'est jamais évoquée. Les besoins spécifiques de protection des migrants ne sont pas pris en compte. Ce silence est d'autant plus troublant que Frontex n'hésite pas par ailleurs à faire des commentaires sur le nombre croissant de "faux demandeurs d'asile " qui tenteraient de franchir les frontières de I'UE, sans se référer à une quelconque méthode lui permettant de distinguer entre "faux " et " vrais " demandeurs d'asile " (Casella Colombeau, Charles, Clochard et Rodier, 2010 : 9).

\footnotetext{
24 Article 26 bis du règlement (UE) $n^{\circ} 1168 / 2011$.

25 Amnesty International European Institutions Office, Caritas Europa, Churches' Commission for Migrants in Europe, Council of Europe, European Asylum Support Office, European Council for Refugees and Exiles (ECRE), European Union Agency for Fundamental Rights (FRA), International Catholic Migration Commission, International Commission of Jurists, International Organization for Migration (OIM), Jesuit Refugee Service (JRS), Organization for Security and Co-operation in Europe, Platform for International Cooperation on Undocumented Migrants (PICUM), Red Cross EU Office, United Nations High Commissioner for Refugees (UNHCR).
}

26 Règlement (CE) n²007/2004 du Conseil portant création d'une Agence européenne pour la gestion de la coopération opérationnelle aux frontières extérieures des États membres de I'Union européenne. 
Pour faire face aux critiques des ONG et d'élus du Parlement européen, un accord est signé le 13 juin 2008 entre Frontex et le $\mathrm{HCR}^{27}$. Mais le rôle des agents onusiens va se limiter à une "sensibilisation " des policiers engagés dans les actions de Frontex pour que ces dernières ne portent pas atteinte aux droits des réfugiés " et des personnes sollicitant une protection internationale, en particulier en ce qui concerne le non refoulement ". Ultérieurement, un des représentants du HCR déclare néanmoins : " nous avons peu d'accès aux opérations conjointes donc nous ne savons pas si les formations que nous menons ont des impacts ou pas " ${ }^{28}$. De son côté, en décembre 2008, le Parlement européen adopte une résolution où il demande "que le mandat de Frontex inclue l'obligation expresse de respecter les normes internationales en matière de droits de I'homme et un devoir de sollicitude envers les demandeurs d'asile lors d'opérations de sauvetage en haute mer ", et invitait à une révision de ce mandat " afin de combler les vides juridiques [...] notamment les conditions juridiques précises des interventions de sauvetage en mer ${ }^{29}$.

La révision du règlement de l'agence adoptée en octobre 2011 va en conséquence présenter quelques évolutions. Elle prévoit pour la première fois des mesures destinées à assurer le respect des droits fondamentaux dans le cadre des activités de Frontex et consacre le principe de non-refoulement. En plus de la création d'un forum consultatif (voir supra) est nommé un officier aux droits fondamentaux dont les recommandations n'ont pas à être suivies obligatoirement par l'agence ${ }^{30}$, et la mise en place d'un code de conduite - juridiquement contraignant - visant à garantir le respect des droits fondamentaux dans toutes les opérations. Le but est pour les gouvernements européens d'assurer " une gestion plus efficace des flux migratoires " selon la formulation bien souvent employée, cependant comme nous allons le constater dans la partie qui suit, derrière l'introduction de ces nouveaux mécanismes, un objectif non avoué demeure : celui de refouler un grand nombre de migrants se présentant aux frontières extérieures de I’UE.

27 Avant la conclusion de cet accord, des liens existaient entre Frontex et le HCR. En 2007, un officier de liaison onusien est présent auprès de l'agence à Varsovie où se situe le siège de Frontex. Cf. Frontex (2008) Rapport annuel 2007, p. 10, [en ligne]. URL : http:// frontex.europa.eu/assets/About_Frontex/Governance_documents/Annual_report/2007/ frontex_general_report_2007_final.pdf

28 Michele Simone, chargé de liaison au Haut Commissariat des Nations unies pour les Réfugiés, lors de l'audition A new mandate for FRONTEX: beyond the security obsessions, a human rights perspective?, organisée par le groupe Verts-ALE au Parlement européen le 15 septembre 2010.

29 Parlement européen, Résolution sur l'évaluation et le développement futur de l'agence Frontex et du système européen de surveillance des frontières Eurosur (2008/2157(INI), 18 décembre 2008.

30 Le 14 novembre 2013, la Médiatrice européenne, Emily O'Reilly, a demandé à Frontex de mettre en place un mécanisme de traitement des plaintes relatives à des violations des droits fondamentaux découlant de son activité. Elle mentionne que "Frontex a rejeté cette recommandation en invoquant le fait que les incidents individuels relèvent de la responsabilité de l'État membre concerné. Ne partageant pas cet avis, Emily O'Reilly a soumis un rapport spécial au Parlement européen, lui demandant son soutien pour persuader Frontex de réviser son approche " (http://www.ombudsman.europa.eu/fr/ press/release.faces/fr/52487/html.bookmark). 


\section{Aux frontières égéennes}

\section{Poséidon : une opération bien réelle}

"Toutes les activités de Frontex se fondent sur le renseignement " ${ }^{31}$ et l'enregistrement d'informations au niveau local, mais inscrites dans le réseau d'analyse des risques de l'agence, intitulé FRAN (Frontex Risk Analysis Network). Ces " analyses de risques " vont donc guider les choix de l'agence pour l'établissement d'opérations temporaires comme celles en mer Égée qui ont été mises en place au cours des étés 2006 et 2007. Selon Frontex, les officiers de liaison envoyés dans cette région par l'Autriche, I'Italie, la Pologne et le Royaume-Uni détectent " un grand nombre de cas d'immigration clandestine et de traite d'êtres humains $"{ }^{32}$ mais ils vont également participer au développement de I'action frontalière.

Depuis 2008, la présence de Frontex en mer Égée ne cesse en effet d'être renforcée autour des îles et à la frontière terrestre entre la Grèce et la Turquie (cf. Carte 2). Si les diverses opérations conjointes dont l'objectif est de " s'opposer aux flux d'immigration clandestine aux frontières maritimes méridionales " ${ }^{33}$ ont été tout d'abord présentées comme temporaires, elles ont au fil du temps un caractère de plus en plus pérenne. En octobre 2010, I'établissement du bureau régional opérationnel dans le port du Pirée suivi dans la foulée du premier déploiement des équipes d'intervention rapide aux frontières (Rapid Border Intervention Team - RABIT) ${ }^{34}$, signe l'établissement dans la région d'une coopération permanente entre les garde-frontières européens. La partie orientale de la Grèce devient un "véritable laboratoire des opérations de l'agence " (FIDH, Migreurop et REMDH, 2014).

Si la quasi-totalité des États membres sont aujourd'hui rassemblés au sein de cette entreprise policière commune en mer Égée ${ }^{35}$, les accords passés entre la Turquie et I'Union européenne ${ }^{36}$ soulignent I'ultime stade ambitionné par Frontex : la coopération avec tous les pays tiers concernés et l'établissement d'activités conjointes menées avec ces derniers. Mais la Turquie ne semble

31 Rapport du Conseil d'administration de Frontex, décision n 1/2008 du 29 janvier 2008 sur la modification 1 du programme de travail 2008, p. 5.

32 Frontex (2007) Rapport annuel 2006 de Frontex, p. 12, [en ligne]. URL : http://frontex. europa.eu/assets/About_Frontex/Governance_documents/Annual_report/2006/annual_ report_20061.pdf

33 Rapport du Conseil d'administration de Frontex, décision n 1/2008 du 29 janvier 2008 sur la modification 1 du programme de travail 2008, p. 5.

34 L'opération a eu lieu à la frontière terrestre gréco-turque dans la région d'Orestiada. Elle a commencé le 2 novembre 2010 pour une période d'un mois, et sera renouvelée jusqu'au 2 mars 2011.

35 L'opération est également destinée à se déployer aux frontières terrestres grecques et bulgares ainsi que dans I'espace maritime entourant l'île de Crète.

36 Memorandum of Understanding between Frontex and the Turkish Ministry of Foreign Affairs, April 2012, [en ligne]. URL : http://www.statewatch.org/observatories_files/ frontex_observatory/MoU\%20Turkey\%2028052012.pdf. Ce mémorandum d'entente qui était en cours d'application lors de la mission effectuée par la FIDH, Migreurop et REMDH (octobre 2013) - prévoit la possibilité d'échanger des informations, d'explorer les possibilités de participer à des opérations de retours conjoints et à des formations sur la gestion des frontières. 
pas pressée d'intégrer ces missions liées en partie aux questions de souveraineté des deux États et aux contentieux historiques, notamment sur les aires maritimes revendiquées par les deux pays.

\section{Push back : violations récurrentes des droits des migrants}

La construction d'un mur de douze kilomètres le long de la rivière Évros, le déploiement de 1800 policiers grecs et l'opération terrestre Poséidon de l'agence Frontex ont drastiquement fait baisser le nombre de migrants interceptés dans la région d'Évros ${ }^{37}$. En conséquence les passages se sont en partie déplacés aux frontières maritimes de la mer Égée ${ }^{38}$ (Frontex, 2012 ; Assemblée Parlementaire du Conseil de l'Europe, 2013). En 2013, selon l'agence, 10427 interceptions ont été effectuées en mer Égée dans le cadre de l'opération Poséidon, ce qui en fait I'un des principaux points d'entrées "irrégulières " sur le territoire européen (FIDH, Migreurop et REMDH, 2014). Mais les interceptions en mer ne sont qu'un des aspects des opérations qui s'y déroulent.

Les garde-côtes grecs sont accusés par les migrants, la société civile et des organisations internationales de défense des droits humains de refouler bien souvent les bateaux arrivant dans les eaux grecques vers les eaux turques et ce, en violation de trois principes reconnus par le droit européen et le droit international.

En renvoyant manu militari les embarcations naviguant dans ses eaux nationales, la Grèce enfreint l'interdiction d'expulsion collective à laquelle elle est tenue par les textes européens ${ }^{39}$. Ces derniers interdisent l'expulsion de groupe de personnes si leur situation individuelle n'a pas été examinée de façon raisonnable et objective. Ainsi, la Grèce, en ne permettant pas aux personnes en recherche de protection internationale de pouvoir accéder à une procédure équitable, viole le droit d'asile consacré par la Convention de Genève relative au statut des réfugiés et le droit européen ${ }^{40}$. Enfin, la Grèce transgresse également le principe de non refoulement prévu aussi dans plusieurs législations ${ }^{41}$ qui interdisent de renvoyer une personne vers son pays d'origine ou un autre pays où sa vie ou sa liberté serait menacée.

Ces refoulements se déroulent loin des regards. Néanmoins des dizaines de témoignages recueillis par Pro-Asyl (2013) et Amnesty International (2013) soulignent que ces renvois se sont inscrits dans la "routine " du contrôle frontalier. Au regard du tableau 1 en annexe, selon les situations enregistrées par

37 De 2000 arrivées par semaine en août 2012, les chiffres sont baissés à dix arrivées par semaine en octobre de la même année.

38102 personnes avaient été arrêtées entre janvier et juillet 2012 à la frontière maritime grecque, 3280 arrestations eurent lieu entre août et décembre de la même année.

39 La convention européenne des droits de I'homme, protocole 4, article 4 et la charte européenne des droits fondamentaux, article 19.1

40 Article 78, Traité de fonctionnement de I'UE ; Article 18, charte des droits fondamentaux ; voir également, Council Directive 2005/85/EC of 1 December 2005 on minimum standards on procedures in Member States for granting and withdrawing refugee status.

41 Article 33 de la Convention de Genève relative au statut des réfugiés. Article 18 de la Charte européenne des droits fondamentaux et l'article 78.1 du Traité de fonctionnement de I'UE (TFUE). 
Pro-Asyl (2013), entre juin 2012 et septembre 2013, plus de 2000 personnes ont été ainsi refoulées. II arrive aussi que les garde-côtes grecs effraient, frappent et volent les migrants lors d'opérations nocturnes. Certains témoignages relatent la présence d'hommes cagoulés et armés qui seraient des agents de forces spéciales grecques. Ces unités interviendraient lorsque les garde-côtes reçoivent l'information selon laquelle les migrants seraient armés. Comment reçoivent-ils cette information, comment la recoupent-ils ? En octobre 2013, interrogeant les autorités grecques à ce sujet, celles-ci ne démentent pas, mais n'ont pas donné davantage de précisions (FIDH, Migreurop et REMDH, 2014).

Lors de ces opérations, des témoignages mentionnent également que les garde-côtes grecs seraient parfois responsables de naufrages de bateaux de migrants en Mer Égée ${ }^{42}$.

L'opération maritime Poséidon coordonnée par Frontex intervient dans un climat particulièrement tendu pour ne pas dire violent. Or depuis 2012, suite à la modification du règlement de l'agence et l'introduction d'une stratégie des droits fondamentaux, tout participant à une opération Frontex a l'obligation de rapporter, dans les plus brefs délais, toutes violations des droits humains dont il a été le témoin ou qu'une personne lui aurait rapportées. Entre décembre 2012 et début 2014, l'agence aurait reçu treize rapports relatant des allégations de violations des droits à la frontière terrestre et maritime grecque : allégations de refoulement, non accès à la procédure d'asile, traitements inhumains et dégradants. Dans deux cas, les violations ont été perpétrées en la présence d'agents de Frontex déployés sur le terrain et dans un cas, un agent a été mis en cause. Pour les autres signalements, les informations ont été collectées par des agents lors d'entretiens avec des migrants (FIDH, Migreurop et REMDH, 2014). L'agence, par le biais de son directeur, peut décider d'arrêter une opération en cas de violations récurrentes et sérieuses. À notre connaissance, elle ne l'a jamais fait.

\section{Les entretiens d'identification (screening) et de récoltes d'informations (débriefing) : de la facilitation du principe de non refoulement aux pratiques intrusives}

Les personnes qui arrivent à accéder au territoire grec sont généralement conduites dans des lieux de privations de libertés, comme des commissariats, des centres de détention administrative (HRW, 2011) ou des centres de premier accueil $^{43}$, établissements où Frontex est également présente. Les policiers des États membres envoyés par l'agence sont là pour identifier des personnes

42 Grèce : les garde-côtes ont-ils provoqué le naufrage du bateau de réfugiés syriens en Mer Égée?, Le courrier des Balkans, vendredi 24 janvier 2014, [en ligne]. URL: http://balkans.courriers.info/article24101.html. Guillot Adéa (2014) Des migrants naufragés accusent la Grèce, Le Monde, vendredi 31 janvier 2014, [en ligne]. URL : http://www.lemonde.fr/europe/article/2014/01/31/des-migrants-naufrages-accusent-lagrece_4357697_3214.html?xtmc=grece_immigration\&xtcr=2

43 Les centres de premiers accueils grecs sont des nouveaux lieux mis en place depuis juin 2013. Ils ont pour fonction de trier les primo arrivants entre ceux qui demandent I'asile et ceux à qui les autorités délivreront une obligation à quitter le territoire grec. Les migrants peuvent être placés dans ces lieux pour une durée maximale de vingt-cinq jours. Selon les textes, les migrants peuvent demander à sortir de l'établissement, mais dans la pratique ces centres s'apparentent à des lieux fermés, soit parce qu'ils sont isolés géographiquement, soit parce qu'ils sont entourés de grillages, barbelés, guérites de surveillance, etc., voire les deux. 
(" screener ") et récolter des informations relatives à l'évolution des routes migratoires (débriefer).

La procédure de screening vise à identifier les nationalités des personnes interceptées afin de faciliter leur renvoi. Selon l'agence, cette action est menée par les policiers grecs accompagnés d'interprètes et d'agents experts en identification, issus de différents États membres. Frontex ne collecterait pas de données lors de ces entretiens, mais la police grecque transfère à l'agence par la suite une partie des informations sans les données personnelles ${ }^{44}$. Frontex soutient donc la police grecque, pendant les entretiens, afin de renforcer leur capacité en termes d'identification.

À I'automne 2013, des allégations de personnes mal identifiées (nationalité ou âge erronés) ont été enregistrées par la FIDH, Migreurop et REMDH. Elles relatent le cas de plusieurs réfugiés palestiniens de Syrie reconnus comme Syriens par le service de premier accueil grec ou comme " apatrides " par la police grecque pendant des entretiens où Frontex était présente. Suite à cette procédure, plusieurs de ces personnes ont reçu une obligation à quitter le territoire d'une durée d'un mois non renouvelable. À ces allégations, Frontex répond que ses agents émettent des avis et des suggestions, les décisions finales sont prises par les autorités grecques. "[Or] Frontex présente pendant les entretiens, ne peut méconnaître cette pratique, [car elle] y participe et facilite par conséquent de possibles violations du principe de non refoulement dans le cadre de I'identification " (FIDH, Migreurop et REMDH, 2014).

Les équipes de débriefing sont également composées de policiers grecs, d'interprètes et d'experts provenant de différents États membres. D'après l'agence, les migrants sont libres d'accepter ou non les entretiens que les équipes proposent. Or, des témoignages recueillis démontrent que les entretiens n'ont pas tous un caractère volontaire. Un témoin n'ayant pas voulu faire I'entretien a été retenu six heures. Face à de telles pratiques, certains migrants se sentent obliger de répondre et appréhendent les suites qui pourraient être données à ces échanges. D'autres mentionnent que durant leur interrogatoire, les policiers les questionnent très précisément sur leurs parcours migratoires, voire l'ensemble de leur vie, de leur naissance à leur arrivée en Grèce. Parfois les migrants pressentent que leurs interlocuteurs ne sont pas grecs, mais l'absence de signes distinctifs, comme les badges d'accréditation de l'agence ${ }^{45}$, ne permet pas aux personnes sujettes à ces entretiens de savoir à qui elles ont affaire. En conséquence ces entretiens deviennent parfois gênants, d'autant que les migrants se trouvent dans une situation de vulnérabilité, confinés dans des lieux privatifs de liberté ${ }^{46}$.

44 À terme, cette procédure devrait être dévolue à la police grecque seule, qui fera remonter les informations collectées à I'agence (FIDH, Migreurop et REMDH, 2014).

45 Le plan Opérationnel Poséidon mer 2012 prévoit que les " débriefeurs " de Frontex n'aient pas à porter leurs uniformes et le brassard bleu de l'agence.

46 Les différents échanges avec l'agence ont montré que celle-ci ne paraît pas encline à fournir plus d'informations autour de ces entretiens ce qui alimente les inquiétudes sur leur déroulement. 


\section{Conclusion}

Sans complètement estomper le rôle prépondérant des États membres formant la frontière extérieure de l'UE, la mise en place d'un mécanisme incitatif comme l'agence Frontex a permis de mutualiser une partie des contrôles migratoires européens. Dans ce processus, "l'autonomisation des praticiens " (Bigo, 1996 : 247) de l'agence n'a cessé de se développer à la fois par l'augmentation de son budget et le renforcement de ses attributions en matière de négociations avec des pays tiers (accords, présence d'agents de liaison européens) alors que les mécanismes de contrôles liés à une législation peu contraignante sont quasiment absents. Ainsi aux frontières extérieures de l'UE, les agents de Frontex sont à la fois des coordinateurs d'opérations conjointes et des acteurs administratifs influençant la prise de décision des polices nationales, voire locales, dans une approche restrictive. En ce sens, la situation migratoire à la frontière orientale de la Grèce rappelle ce que Casella Colombeau (2013 : 524) évoque à propos de la situation sur les côtes méridionales espagnoles où elle montre "que la régularité des interventions de l'agence aux frontières maritimes avait des effets sur la juridiction de la Police des frontières espagnoles, notamment vis-à-vis de la Guardia Civil ". Par ailleurs, Frontex essaie également d'élargir son champ d'intervention bien au-delà des frontières extérieures pour anticiper et renforcer les opérations de contrôles des migrations faisant des pays voisins à I'UE un espace-tampon. Dans ces zones de transition comme celles qui se situent de part et d'autre de la frontière gréco-turque, les limites demeurent donc invisibles, voire imperceptibles - $d^{\prime}$ autant plus quand les États limitrophes sont liés par des contentieux territoriaux - engendrant bien souvent des pratiques policières arbitraires permettant de refouler plus facilement nombre de migrants.

\section{Références bibliographiques}

Amnesty International (2013) Frontier Europe: Human rights abuses on Greece's border with Turkey, 40 p., [en ligne]. URL : http://www.amnesty.org/ en/library/asset/EUR25/008/2013/en/d93b63ac-6c5d-4d0d-bd9f-ce2774c84ce7/ eur250082013en.pdf

Anderson Malcom and Den Boer Monica (Eds.) (1994) Policing across national boundaries, London-New York, Pinter Publishers, 204 p.

Assemblée Parlementaire du Conseil de l'Europe (2013) Migrations et asile : montée des tensions en Méditerranée orientale, 19 p., [en ligne]. URL : http://www. assembly.coe.int/nw/xml/XRef/X2H-Xref-ViewPDF.asp?FilelD=19349\&lang=fr

Bigo Didier (Éd.) (1998) Sécurité et immigration, Cultures \& Conflits, 31-32, 135 p.

Bigo Didier (1996) Polices en réseau. L'expérience européenne, Paris, Presses de Sciences Po., $356 \mathrm{p}$.

Busch Heiner (2002) Le piège légaliste. La coopération policière après Tampere, Cultures \& Conflits, 45, pp. 125-132.

Casella Colombeau Sara (2013) Surveiller les personnes, garder les frontières, définir le territoire. La Police Aux Frontières après la création de l'espace Schengen (1953-2004), Thèse de doctorat en science politique, $598 \mathrm{p}$. 
Casella Colombeau Sara, Charles Marie, Clochard Olivier et Rodier Claire (2010) Agence Frontex : quelles garanties pour les droits de l'Homme ? Etude sur l'Ágence européenne aux frontières extérieures en vue de la refonte de son mandat (Étude réalisée sous la direction des eurodéputées Hélène Flautre, Franziska Keller et Barbara Lochbihler), 48 p.

Casella Colombeau Sara et Clochard Olivier (2012) Officiers de liaison : ingérence et " coopération " au service du contrôle migratoire, in Migreurop, Atlas des migrants en Europe. Géographie critique des politiques migratoires, Paris, Armand Colin, pp. 44-47.

Drapier Christian (2011) Frontex dans le contexte de la situation à la frontière gréco-turque, Ceriscope Frontières, [en ligne]. URL : http://ceriscope.sciencespo.fr/content/part2/frontex-dans-le-contexte-de-la-situation-a-la-frontiere-grecoturque-\%3Fpage\%3Dshow

FIDH, Migreurop et REMDH (2014) Frontex : entre Grèce et Turquie, la frontière du déni, 98 p., [en ligne]. URL : http://www.migreurop.org//MG/pdf/rapport_fr_ grece_turquie_site_3_-_copie-3.pdf

Frontex (2013) General Report 2012, 72 p., [en ligne]. URL : http://frontex. europa.eu/assets/About_Frontex/Governance_documents/Annual_report/2012/ EN_General_Report_2012.pdf

Human Rights Watch (2011) The EU's dirty hands, 64 p., [en ligne]. URL : http:// www.hrw.org/sites/default/files/reports/greece0911webwcover_0.pdf

Jeandesboz Julien (2012) Contrôles aux frontières de l'Europe. Frontex et I'espace Schengen, La vie des idées.fr, 12 p., [en ligne]. URL : http://www.laviedesidees.fr/Controles-aux-frontieres-de-I.html

Lévy René et Dominique Monjardet (2002) Les polices nationales et l'unification européenne, enjeux et interactions. Remarques introductives, Cultures \& Conflits, 48 , pp. 5-14.

Maguer Azilis (2002) La coopération policière transfrontalière, moteur de transformations dans I'appareil de sécurité français, Cultures \& Conflits, 48, pp. 33-56.

Migreurop (2012) L'atlas des migrants en Europe. Géographie critique des politiques migratoires, Paris, Armand Colin, $144 \mathrm{p}$.

Peers Steve (2014) New EU rules on maritime surveillance: will they stop the deaths and push-backs in the Mediterranean?, 7 p., [en ligne]. URL : http://www. statewatch.org/analyses/no-237-maritime-surveillance.pdf

Pro-Asyl (2013) Pushed Back - Systematic human rights violations against Refugees in the Aegean Sea and the Greek-Turkish land border, 76 p., [en ligne]. URL : http://www.proasyl.de/fileadmin/fm-dam/I_EU_Fluechtlingspolitik/proasyl_ pushed_back_24.01.14_a4.pdf

Rodier Claire (2011) Frontex, la petite muette, Vacarme, 55, pp. 36-39, [en ligne]. URL : http://www.vacarme.org/article2015.html

Rodier Claire (2010) Frontex, I'agence tout risque, Plein Droit, 87, pp. 8-11.

Sabatier Magali (2001) La coopération policière européenne, Paris, L'Harmattan, $510 \mathrm{p}$.

Sheptycki James (1997) Faire la police dans la Manche : I'évolution de la coopération transfrontalière (1968-1996), Cultures \& Conflits, 26-27, pp. 93-121, [en ligne]. URL : www.conflits.org/document367.html 


\section{Annexe}

Tableau 1 : Nombre total de personnes refoulées selon les données de recherche

TABLE 1.

Total of pushed back persons according to the research data

\begin{tabular}{|c|c|c|c|c|}
\hline No. & Date of p.b. & Place of $p . b$. & $\begin{array}{l}\text { Nationality of } \\
\text { person/s int. }\end{array}$ & $\begin{array}{l}\text { No. of person/s } / \mathrm{s} \\
\text { pushed back }\end{array}$ \\
\hline 1 & 12-Jul-13 & Evros & Afghanistan & \\
\hline 2 & 07-Jul-13 & Evros & & $x^{*}(24)$ \\
\hline 3 & July 2013 & Evros & Afghanistan & 14 \\
\hline 4 & 17-Jul-13 & Evros & Eritrea & 25 \\
\hline 5 & June 2013 & Evros & Afghanistan & 24 \\
\hline 6 & June 2013 & Evros & Eritrea & \\
\hline 7 & May 2013 & Evros & Eritrea & $x^{22}+241$ \\
\hline 8 & $02-$ May-13 & $\begin{array}{l}\text { Evros } \\
\text { Evros }\end{array}$ & Somalia & \\
\hline 9 & April 2013 & Evros & Afghanistan & $100-200$ \\
\hline 10 & October 2012 & Evros & Afghanistan & \\
\hline 11 & October 2012 & Evros & Afghanistan & 13 \\
\hline 12 & September 2012 & Evros & Afghanistan & 15 \\
\hline 13 & September 2012 & Evros & Afghanistan & \\
\hline 14 & August 2012 & Evros & Afghanistan & $x^{*}(24)$ \\
\hline 15 & August 2012 & Evros & Syria & $40-50$ \\
\hline 16 & August 2012 & Evros & Syria & 13 \\
\hline 17 & June 2012 & Evros & Syria & \\
\hline 18 & December 2011 & Evros & Afghanistan & $X^{*}(24)$ \\
\hline 19 & $08-$ Sep-13 & $\begin{array}{l}\text { Lesyos } \\
\text { Lesyos }\end{array}$ & $\begin{array}{l}\text { Syria } \\
\text { Systan }\end{array}$ & \\
\hline & August 2013 & Lesyos & Afghanistan & 48 \\
\hline 21 & August 2013 & $\begin{array}{l}\text { Lesvos } \\
\text { Lestos }\end{array}$ & Afghanistan & 48 \\
\hline 22 & 13-Jul-13 & Lesvos & Afghanistan & 30 \\
\hline 23 & 08-May-13 & $\begin{array}{l}\text { Lesyos } \\
\text { Lestos }\end{array}$ & Somalia & 45 \\
\hline 24 & Spring 2013 & $\begin{array}{l}\text { Lesvos } \\
\text { Lestos }\end{array}$ & Eritrea & 45 \\
\hline 25 & Spring 2013 & Lesros & Eritrea & \\
\hline & Spring 2013 & Lesvos & Eritrea & $X *[35]$ \\
\hline 27 & May 2013 & Lesvos & Somalia & 25 \\
\hline 28 & April 2013 & Lesvos & Afghanistan & 30 \\
\hline 29 & March 2013 & Lesvos & Afghanistan & 30 \\
\hline 30 & January 2013 & $\begin{array}{l}\text { Lesyos } \\
\text { Lestos }\end{array}$ & Afghanistan & 30 \\
\hline 31 & January 2013 & & Afghanistan & \\
\hline $\begin{array}{l}31 \\
32\end{array}$ & Dezember 2012 & $\begin{array}{l}\text { Lesyos } \\
\text { Lesios }\end{array}$ & $\begin{array}{l}\text { Argnamistan } \\
\text { Afghanistan }\end{array}$ & $\begin{array}{l}28 \\
42\end{array}$ \\
\hline 33 & October 2012 & Lesvos & Afghanistan & 45 \\
\hline & 22 -Sep-13 & Samos & Syria & \\
\hline 35 & 28-Aug-13 & Samos & Syria & $X^{* *}[35]$ \\
\hline & 22-Aug- 13 & Samos & Syria & 40 \\
\hline 37 & 22-Aug- 13 & Samos & Syria & 50 \\
\hline
\end{tabular}

\begin{tabular}{|c|c|c|c|c|}
\hline No. & Date of p.b. & Place of p.b. & $\begin{array}{l}\text { Nationality of } \\
\text { person } / s \text { int. }\end{array}$ & $\begin{array}{l}\text { No. of person/s } \\
\text { pushed back }\end{array}$ \\
\hline 38 & 11-Aug-13 & Samos & Syria & 40 \\
\hline 39 & 04-Aug-13 & Samos & Syria & 42 \\
\hline 40 & August 2013 & Samos & Syria & $30-40$ \\
\hline 41 & August 2013 & Samos & Syria & \\
\hline 42 & $30-J u l-13$ & Samos & Syria & 40 \\
\hline 43 & 28-Jul-13 & Samos & France & 28 \\
\hline 44 & $05-J u l-13$ & Samos & Afghanistan & 45 \\
\hline 45 & July 2013 & Samos & Afghanistan & 33 \\
\hline 46 & June 2013 & Samos & Syria & 47 \\
\hline 47 & June 2013 & Samos & Syria & 20 \\
\hline & June 2013 & Samos & & 18 \\
\hline 49 & Spring 2013 & Samos & Eritrea & 46 \\
\hline 50 & 27-Aug-13 & Chios & Syria & 6 \\
\hline 51 & 18-Aug-13 & Chios & Syria & $X^{* *}[35]$ \\
\hline 52 & August 2013 & Chios & Syria & 30 \\
\hline 53 & 26-Aug-13 & Farmakonisi & Syria & 14 \\
\hline 54 & 17-Aug-13 & Farmakonisi & Syria & \\
\hline 55 & 08-Aug-13 & Farmakonisi & Syria & 46 \\
\hline 56 & 10-Jul-13 & unspecified island & Afghanistan & 40 \\
\hline 57 & 10-Jul-13 & unspecified island & Afghanistan & \\
\hline 58 & $05-J u l-13$ & unspecified island & Afghanistan & $\mathrm{X}^{* *}[35]$ \\
\hline 59 & summer 2013 & unspecified island & Syria & 61 \\
\hline & summer 2013 & unspecified island & Syria & 45 \\
\hline 61 & November 2012 & unspecified island & Syria & 24 \\
\hline
\end{tabular}

* There were four instances where the actual number of people pushed back from Evros was not mentioned, yet we estimate based on our data that on an average approximately 24 persons per time are

(the Aegean was "The wer

According to the statistics based on the research data in the most conservative interpretation of the numbers given by the interviewees, 2.035 persons were affected by push-backs only based on the incidents reported here for the given period.

175 | We did not include the number 100 las it occurred only once and seemed to be an exception) in

Source : Pro-Asyl (2013) Pushed Back - Systematic human rights violations against Refugees in the Aegean Sea and the Greek-Turkish land border, Frankfurt, Pro-Asyl, pp. 72-73. 


\section{Eva Ottavy et Olivier Clochard}

\section{Franchir les dispositifs établis par Frontex. Coopérations policières transfrontalières et refoulements en mer Égée}

Depuis le milieu des années 2000, les gouvernements européens ont mis en place une agence - Frontex - visant à coordonner et mutualiser les opérations de surveillance aux frontières extérieures de I'Union européenne. Un de ses principaux objectifs est de lutter contre l'immigration dite irrégulière. En s'intéressant au durcissement des contrôles migratoires et notamment à ceux qui sont développés dans la région de la mer Égée, à la frontière entre la Grèce et la Turquie, il s'agit de montrer la façon dont des collaborations policières et leurs dispositifs se sont développés pour comprendre l'effort que les migrants " irréguliers " doivent fournir pour les contourner et accéder au territoire de l'Union ; depuis 2006, une des pratiques des policiers aux frontières est de refouler bien souvent les migrants vers la Turquie sans que ces derniers puissent faire valoir leurs droits.

\section{Getting around Frontex Controls. Cross-border Police Cooperation and Refoulement in the Aegean}

In the mid-2000s, European governments established a European agency Frontex - to coordinate and pool surveillance operations conducted at the external borders of the European Union. One of the main objectives of this agency is to fight so-called illegal immigration. By looking at the tightening of migration controls, especially those implemented in the Aegean region on the border between Greece and Turkey, the authors want to show the way in which police cooperation and mechanisms have developed in order to understand the efforts involved on the part of "irregular" migrants to circumvent them and gain access to EU territory. Because since 2006, border police officers have very often pushed back migrants to Turkey without the possibility of exercising their rights.

\section{.. Cruzar los controles establecidos por Frontex. La cooperación policial transfronteriza y represiones en el Egeo}

Desde mediados de la década de 2000, los gobiernos europeos han creado una agencia - Frontex - con el fin de coordinar y mutualizar las operaciones de vigilancia de las fronteras exteriores de la Unión Europea. Uno de sus principales objetivos es luchar contra la llamada inmigración ilegal. Al observar el endurecimiento de los controles de migración, particularmente los que se desarrollan en la región del mar Egeo, en la frontera entre Grecia y Turquía, se quiere poner de relieve de qué manera se desarrollan las colaboración policiales y sus dispositivos para comprender el esfuerzo de los inmigrantes «irregulares» para lograr eludirlos y poder acceder al territorio de la UE; desde 2006, una de las prácticas de los agentes de policía de fronteras es la de empujar a los migrantes hacia Turquía, sin que puedan hacer valer sus derechos. 